\title{
Information on in- and out-of-plane correlated roughness in multilayers from $\mathrm{x}$-ray specular reflectivity
}

\author{
V P Romanov ${ }^{1}$, S V Ulyanov ${ }^{1}$, V M Uzdin ${ }^{1}$, G Nowak ${ }^{2}$, M Vadala ${ }^{2}$ and \\ H Zabel ${ }^{2}$ \\ ${ }^{1}$ St Petersburg State University, Ul'yanovskaya ul.1, Petrodvorets, St Petersburg 198904, Russia \\ ${ }^{2}$ Department of Physics, Ruhr-University Bochum, D-44780 Bochum, Germany
}

Received 1 February 2008, in final form 30 March 2008

Published 8 May 2008

Online at stacks.iop.org/JPhysD/41/115401

\begin{abstract}
Specular reflectivity measurements of artificial multilayers often exhibit a broadening of high order Bragg peaks and an intensity drop off that is slower than expected from the $q^{-4}$ Fresnel dependence modified with a static Debye-Waller factor accounting for the interfacial roughness. This behaviour is described on the basis of a model that takes into account the finite size of the detector slit, which determines the instrumental resolution. The amplitude of the interface roughness as well as the in- and out-of-plane correlation lengths strongly affect the shape of the specular x-ray spectra. Information about in-plane and out-of-plane correlated roughness can be obtained, therefore, not only from diffuse scattering data but also from the quasi-specular reflectivity by relaxing the instrumental resolution. Moreover, for systems with short correlation lengths, quasi-specular reflectivity scans are more informative than rocking curves across Bragg reflections. The model is applied to the interpretation of x-ray scattering results on two multilayer systems: $\mathrm{MgO}\left(\begin{array}{lll}0 & 0 & 1\end{array}\right) /[\mathrm{Fe}(2 \mathrm{ML}) / \mathrm{V}(16 \mathrm{ML})]_{30}$ and

$\mathrm{Si} / \mathrm{SiO}_{2} /[\mathrm{CoFeB}(3 \mathrm{~nm}) / \mathrm{MgO}(1.5 \mathrm{~nm})]_{15}$. The simulation of the experimental reflectivity scans properly describes the broadening of the higher order Bragg peaks, the damping of Kiessig fringes, and the increased intensity at higher scattering vectors as compared with the purely specular reflectivity.
\end{abstract}

(Some figures in this article are in colour only in the electronic version)

\section{Introduction}

$\mathrm{X}$-ray reflectivity (XRR) has been proven to be a very powerful tool for the structural analysis of thin films and superlattices. It is widely used for determining film thicknesses, multilayer periods and interface roughness of solid and soft matter material layers. The multitude of applications in different fields has recently been summarized in a brief review by Bontempi et al [1]. Further references can be found in the proceedings of the international conference on surface $\mathrm{x}$-ray and neutron scattering; the proceedings of the last one in this series is published in [2]. Although the method is popular and widespread, there are some limitations that one should consider in order to extract reliable structural parameters from fits to the experimental curves. One general rule of thumb is that the interface roughness should not be too large, i.e. less than about $5 \mathrm{~nm}$, and that the roughness should not exceed the layer thickness. Nonetheless, occasionally one obtains disturbing experimental results, which cannot be described by standard reflectivity models. One particular example is multilayers where the first few Bragg reflections are well behaved, but higher order Bragg reflections are broadened beyond reasonable instrumental resolution and have intensities that are higher than expected from a Debye-Waller type decay due to interface roughness. In this contribution we will discuss reasons for this behaviour and provide a model which can describe with a reasonable amount of accuracy well-defined multilayers with smooth interfaces as well as illdefined multilayers with rough interfaces.

There are a number of models and fitting routines that are either circulated among the practitioners of XRR, published and free for download [3,4] or commercially available [5]. 
In addition, a new model has recently been announced for the fitting of reflectivity curves, which is quoted as the BedeMercury model [6]. It consists of a self-correcting recursive mode, which provides structural parameters for each individual layer and interface and which usually fits the experimental data very well. In contrast to this approach, our approach described here tries to keep the number of parameters at a minimum level while providing physical meaningful numbers for the roughness, the in- and out-of-plane correlation lengths, the jaggedness of the interfaces, etc. In this respect it follows the seminal description of Sinha et al [7] with the extension to multilayers by Sanyal et al [8]. An updated account on reflectivity models has been provided by Pietsch et al in a monograph [9].

This paper is organized as follows: in section 2 we provide a theoretical description for the XRR of multilayers, the procedure of sample preparation and $\mathrm{x}$-ray measurements are described in section 3 and section 4 we discuss the obtained results.

\section{Theoretical description}

We first consider the elastic x-ray scattering from periodic multilayers containing $N$ metallic bilayers on a substrate with randomly rough interfaces. The differential cross section $\mathrm{d} \sigma / \mathrm{d} \Omega$ for $\mathrm{x}$-ray scattering from this system is then given in the first Born approximation by [7]

$\frac{\mathrm{d} \sigma}{\mathrm{d} \Omega}=\frac{k_{0}^{4}}{(4 \pi)^{2}}\left\langle\int_{V} \int_{V} \mathrm{~d} \boldsymbol{r}_{1} \mathrm{~d} \boldsymbol{r}_{2} \Delta\left(\boldsymbol{r}_{1}\right) \Delta\left(\boldsymbol{r}_{2}\right) \mathrm{e}^{-\mathrm{i} \boldsymbol{q}\left(\boldsymbol{r}_{1}-\boldsymbol{r}_{2}\right)}\right\rangle$,

where $k_{0}=2 \pi / \lambda$ is the wave number, $\lambda$ is the wavelength of the radiation, $\boldsymbol{q}=\boldsymbol{k}_{r}-\boldsymbol{k}_{0}$ is the scattering vector, $\boldsymbol{k}_{r}$ and $\boldsymbol{k}_{0}$ are the wave vectors of the scattered and the incident waves, respectively. The bracket $\langle\cdots\rangle$ implies a statistical ensemble average and $V$ is the scattering volume. The notation $\Delta(\boldsymbol{r})$ is used for the deviation of the permittivity $\varepsilon(\boldsymbol{r})$ from the vacuum value. Neglecting the imaginary part of $\varepsilon(\boldsymbol{r})$ we have

$$
\varepsilon(\boldsymbol{r})=1-\Delta(\boldsymbol{r}) .
$$

The use of the first Born approximation instead of the distorted wave Born approximation (DWBA) is justified if the angle between the incident (scattered) beam and the sample surface is much larger than the critical angle of total external reflection.

The surface of the substrate is assumed to be slightly displaced from the plane $z=0$ and $z_{0}\left(\boldsymbol{r}_{\perp}\right)$ is the height of the surface above the plane $z=0$ at the point with radius vector $\boldsymbol{r}_{\perp}=(x, y)$. The substrate is assumed to occupy the half space $z<z_{0}\left(\boldsymbol{r}_{\perp}\right)$. Each bilayer consists of two layers with different electron densities and $z_{n}\left(\boldsymbol{r}_{\perp}\right), n=1,2, \ldots, 2 N-1$ is the height of the $n$th rough interface above the plane $z=0$, whereas $z_{2 N}\left(\boldsymbol{r}_{\perp}\right)$ is the height of the topmost boundary of the multilayer. The perpendicular location of the $n$th layer is thus defined by the inequalities

$$
z_{n-1}\left(\boldsymbol{r}_{\perp}\right)<z<z_{n}\left(\boldsymbol{r}_{\perp}\right), \quad n=1,2, \ldots, 2 N .
$$

If the permittivity $\varepsilon(\boldsymbol{r})$ is constant inside each layer, then after integration of equation (1) in the $z$-direction we find the following expression for $\mathrm{x}$-ray scattering on multilayer structures [7, 10-12]:

$$
\begin{aligned}
\frac{\mathrm{d} \sigma}{\mathrm{d} \Omega} & =\frac{k_{0}^{4}}{(4 \pi)^{2} q_{z}^{2}} \sum_{n, m=0}^{2 N} \Delta \varepsilon_{n} \Delta \varepsilon_{m} \int_{S} \int_{S} \mathrm{~d} \boldsymbol{r}_{\perp 1} \mathrm{~d} \boldsymbol{r}_{\perp 2} \mathrm{e}^{-\mathrm{i} \boldsymbol{q}_{\perp} \cdot\left(\boldsymbol{r}_{\perp 1}-\boldsymbol{r}_{\perp 2}\right)} \\
& \times\left\langle\mathrm{e}^{-\mathrm{i} q_{z}\left(z_{n}\left(\boldsymbol{r}_{\perp 1}\right)-z_{m}\left(\boldsymbol{r}_{\perp 2}\right)\right)}\right\rangle
\end{aligned}
$$

where $\boldsymbol{q}=\left(\boldsymbol{q}_{\perp}, q_{z}\right)$ and $\Delta \varepsilon_{n}$ is the jump of the permittivity at the $n$th interface. Representing the height of the interface $z_{n}\left(\boldsymbol{r}_{\perp}\right)$ above the plane $z=0$ as a sum of the mean value $z_{n}$ and the deviation $h_{n}\left(\boldsymbol{r}_{\perp}\right)$ :

$$
z_{n}\left(\boldsymbol{r}_{\perp}\right)=z_{n}+h_{n}\left(\boldsymbol{r}_{\perp}\right)
$$

and assuming that $h_{n}\left(\boldsymbol{r}_{\perp}\right)$ is a Gaussian random variable, we obtain

$$
\begin{aligned}
\frac{\mathrm{d} \sigma}{\mathrm{d} \Omega} & =\frac{k_{0}^{4}}{(4 \pi)^{2} q_{z}^{2}} \sum_{n, m=0}^{2 N} \Delta \varepsilon_{n} \Delta \varepsilon_{m} \mathrm{e}^{-\mathrm{i} q_{z}\left(z_{n}-z_{m}\right)} \mathrm{e}^{\left[-\frac{q_{z}^{2}}{2}\left(\left\langle h_{n}^{2}\right\rangle+\left\langle h_{m}^{2}\right\rangle\right)\right]} \\
& \times\left\{S(2 \pi)^{2} \delta\left(q_{x}\right) \delta\left(q_{y}\right)+\int_{S} \int_{S} \mathrm{~d} \boldsymbol{r}_{\perp 1} \mathrm{~d} \boldsymbol{r}_{\perp 2} \mathrm{e}^{-\mathrm{i} q_{\perp} \cdot\left(\boldsymbol{r}_{\perp 1}-\boldsymbol{r}_{\perp 2}\right)}\right. \\
& \left.\times\left(\mathrm{e}^{\left[q_{z}^{2}\left\langle h_{n}\left(\vec{r}_{\perp 1}\right) h_{m}\left(\vec{r}_{\perp 2}\right)\right\rangle\right]}-1\right)\right\},
\end{aligned}
$$

where $\delta(q)$ is the $\delta$-function. For periodic multilayers with the structural period $D$ in the $z$-direction and mean upper layer thickness $d$ in each bilayer we have

$z_{0}=0, \quad z_{2 k}=k D, \quad z_{2 k-1}=k D-d ;$

$$
k=1,2, \ldots, N \text {. }
$$

The specular reflected component with $\boldsymbol{q}_{\perp}=0$ is separated out in equation (6). If the interface roughness is uncorrelated at different points in the $(x, y)$ plane:

$$
\left\langle h_{n}\left(\boldsymbol{r}_{\perp 1}\right) h_{m}\left(\boldsymbol{r}_{\perp 2}\right)\right\rangle=0, \quad\left|\boldsymbol{r}_{\perp 1}-\boldsymbol{r}_{\perp 2}\right| \neq 0,
$$

then the integral term in equation (6) does not produce any contribution to the scattered wave. For the roughnessroughness correlation function the following expression was proposed in [13]:

$$
\begin{aligned}
& \left\langle h_{n}\left(\boldsymbol{r}_{\perp 1}\right) h_{m}\left(\boldsymbol{r}_{\perp 2}\right)\right\rangle=\sigma_{n} \sigma_{m} \exp \left[-\left(\frac{\left|z_{n}-z_{m}\right|}{l_{z n, m}}\right)^{s}\right] \\
& \times \exp \left[-\left(\frac{\left|\boldsymbol{r}_{\perp 1}-\boldsymbol{r}_{\perp 2}\right|}{l_{\perp n, m}}\right)^{2 H_{n, m}}\right],
\end{aligned}
$$

where $s=1$ or 2 , and the Hearst parameters $0 \leqslant H_{n m} \leqslant 1$ are determined by various types of roughnesses, $l_{\perp n, m}$ and $l_{z n, m}$ are the in- and out-of-plane correlation lengths and $\sigma_{n}$ is the mean square roughness of the $n$th interface. In what follows we assume that the roughness is the same for all $n$ interfaces in the multilayer, i.e. $\sigma_{n}=\sigma$; furthermore we set $l_{z n, m}=l_{z}$; $l_{\perp n, m}=l_{\perp} ; H_{n, m}=H=1 ; s=2$. The cross section of the $\mathrm{X}$-ray scattering $\Delta \sigma$, which is collected over the solid angle $\Delta \Omega$ can then be written as

$$
\Delta \sigma=\int_{\Delta \Omega}\left(\frac{\mathrm{d} \sigma}{\mathrm{d} \Omega}\right) \mathrm{d} \Omega .
$$


Here the differential of the solid angle $\mathrm{d} \Omega$ can be expressed through the differentials of the scattering vector [7]

$$
\mathrm{d} \Omega=\frac{\mathrm{d} q_{x} \mathrm{~d} q_{y}}{k_{0}^{2} \sin \theta}
$$

where $\theta$ is the glancing angle to the surface.

For the reflection coefficient $R$, which is defined as the ratio of the radiation scattered into the solid angle $\Delta \Omega$ to the intensity of the incident radiation, we find

$$
R=\frac{1}{S \sin \theta} \int_{\Delta \Omega}\left(\frac{\mathrm{d} \sigma}{\mathrm{d} \Omega}\right) \mathrm{d} \Omega=\frac{2 k_{0}}{S q_{z}} \Delta \sigma,
$$

where the differential cross section $(\mathrm{d} \sigma / \mathrm{d} \Omega)$ is given by equation (6). It is convenient to present the reflectivity as a sum of two terms

$$
R=R_{1}+R_{2}
$$

where

$$
R_{1}=\frac{k_{0}^{4}}{q_{z}^{4}} \sum_{n, m=0}^{2 N} \Delta \varepsilon_{n} \Delta \varepsilon_{m} \mathrm{e}^{-\mathrm{i} q_{z}\left(z_{n}-z_{m}\right)} \mathrm{e}^{-q_{z}^{2} \sigma^{2}}
$$

and

$$
\begin{aligned}
R_{2}= & \frac{k_{0}^{5}}{4 \pi q_{z}^{3}} \sum_{n, m=0}^{2 N} \Delta \varepsilon_{n} \Delta \varepsilon_{m} \mathrm{e}^{-\mathrm{i} q_{z}\left(z_{n}-z_{m}\right)} \mathrm{e}^{-q_{z}^{2} \sigma^{2}} \\
& \times \int_{\Delta \Omega} \mathrm{d} \Omega \int_{0}^{\infty} r_{\perp} \mathrm{d} r_{\perp} J_{0}\left(q_{\perp} r_{\perp}\right)\left(\mathrm{e}^{\left[q_{z}^{2}\left\langle h_{n}\left(r_{\perp}\right) h_{m}(0)\right\rangle\right]}-1\right) .
\end{aligned}
$$

Here $J_{0}(x)$ is the zero-order Bessel function. $R_{1}$ is usually referred to as the specular reflectivity.

The value $R_{2}$ contains information about the spatial roughness correlation not included in $R_{1}$. It depends on both $q_{z}$ and $q_{\perp}$. For finite $\Delta \Omega, R_{2}$ contributes both to diffuse as well as to specular scattering. If $R_{2}$ is considered as a function of $q_{\perp}$ for fixed $q_{z}$ corresponding to one of the Bragg peaks, it describes the transverse lineshape usually referred to as rocking curve of that particular Bragg peak. For the description of specular scattering, $R_{2}$ has to be regarded as a function of $q_{z}$, whereas $q_{\perp}$ has to be taken close to zero.

First we discuss the diffuse scattering. It is often thought that from diffuse scattering the in-plane correlation length $l_{\perp}$ can be determined. However this is true only if $l_{\perp}$ lies within the defined limits. Indeed usually in experiments on diffuse scattering the orientation of the sample varies at fixed scattering angle $2 \theta$. If the measurements are performed near the $n$th Bragg peak, then $2 \theta=2 \theta_{n}$. The available interval for the rocking angle $|\omega|<\theta_{n}$ determines the limitation on the transverse component of the scattering vector,

$$
q_{\perp}<\frac{n^{2} \pi \lambda}{D^{2}}
$$

As an example, for $\lambda \approx 0.15 \mathrm{~nm} ; D \approx 4 \mathrm{~nm}$ we get $q_{\perp} \leqslant 0.03 n^{2} \mathrm{~nm}^{-1}$, which is a rather limited range for $q_{\perp}$.

As seen from equation (15) the slope of the diffuse scattering curve is noticeable only for correlation

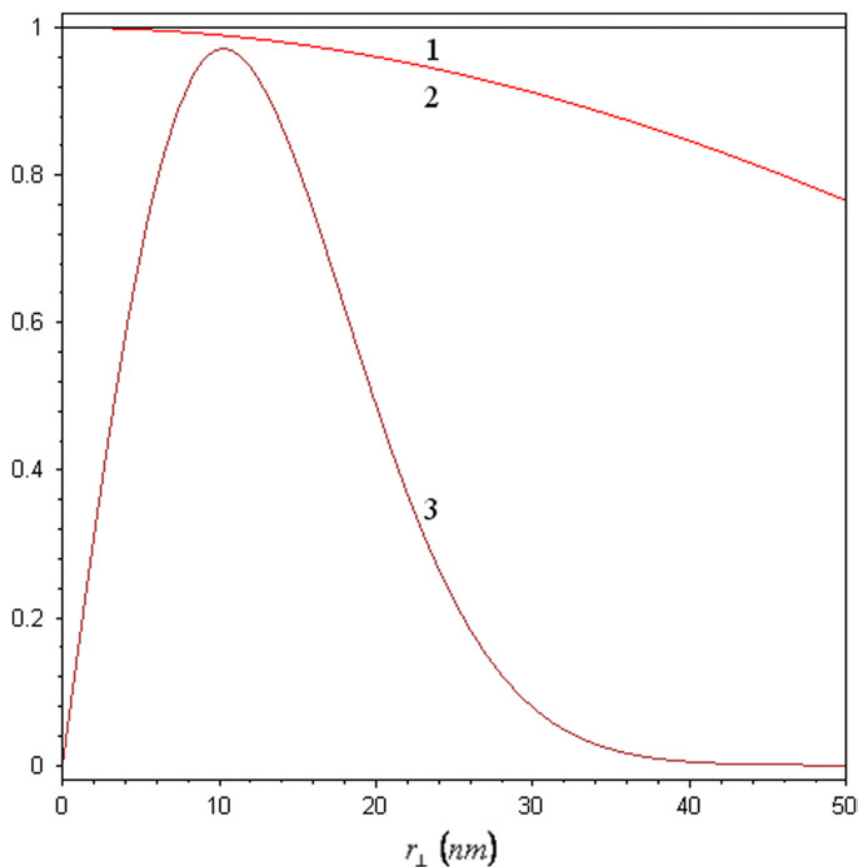

Figure 1. Dependences of factors $I_{1}$ and $I_{2}$ on $r_{\perp}$. Curves (1) and (2) represent $I_{1}\left(r_{\perp}\right)$ for $q_{\perp}=10^{-3} \mathrm{~nm}^{-1}$ and $q_{\perp}=2 \times 10^{-2} \mathrm{~nm}^{-1}$, respectively. Curve (3) is $0.7 \cdot I_{2}\left(r_{\perp}\right)$ for $\sigma=0.3 \mathrm{~nm}, l_{\perp}=15 \mathrm{~nm}$.

lengths, which obey the condition $q_{\perp} l_{\perp} \geqslant 1$, because only in this case the oscillations of the Bessel function in equation (15) are essential.

To illustrate the dependence of the two factors in the integrand of equation (15), $I_{1}\left(r_{\perp}\right)=J_{0}\left(q_{\perp} r_{\perp}\right)$ and $I_{2}=\left(\mathrm{e}^{\left[q_{z}^{2}\left\langle h_{n}\left(r_{\perp}\right) h_{m}(0)\right\rangle\right]}-1\right)$ are plotted in figure 1 as a function of $r_{\perp}$. For small correlation lengths in regions where $I_{2}$ is not small, the Bessel function is almost constant and close to 1 in the whole experimentally achievable interval of $q_{\perp}$ variation. Therefore the diffuse scattering does not depend on the rocking angle for small correlation lengths. If rocking curves are measured near the $n$th Bragg peak, correlation lengths which fulfil $l_{\perp} \leqslant 30 / n^{2} \mathrm{~nm}$ cannot be determined from the diffuse scattering data.

For very large $l_{\perp}$, when $q_{\perp} l_{\perp} \gg 1$, the information about $l_{\perp}$ is lost because of the oscillations that appear in the Bessel function, as shown in figure 2 . If in the same $l_{\perp}$ interval the Bessel function has several oscillations, the contribution to the integral from the tail of $I_{2}$ is rather small. But it is this tail which contains the information about the in-plane correlation length. Therefore a change in $l_{\perp}$ will not affect $R_{2}$ if $q_{\perp} l_{\perp} \gg 1$ is fulfilled. From this we conclude that the diffuse scattering data allow a determination of $l_{\perp}$ only for values that obey $q_{\perp} l_{\perp} \cong 1$.

Similarly to the diffuse scattering, the specular scattering data also contain information about interface roughness due to contributions from both $R_{1}$ and $R_{2}$. The term $R_{1}$ includes a static Debye-Waller like factor which does not depend on the roughness correlations. The $R_{2}$ term depends on the geometrical structure via the oscillating term $\mathrm{e}^{-\mathrm{i} q_{z}\left(z_{n}-z_{m}\right)}$, on the roughness magnitude via the factor $\mathrm{e}^{\left[-\left(q_{z}^{2} / 2\right)\left(\sigma_{n}^{2}+\sigma_{m}^{2}\right)\right]}$ and on the correlation lengths $l_{\perp} l_{z}$ via the correlation function $\left\langle h_{n}\left(r_{\perp}\right) h_{m}(0)\right\rangle$. 


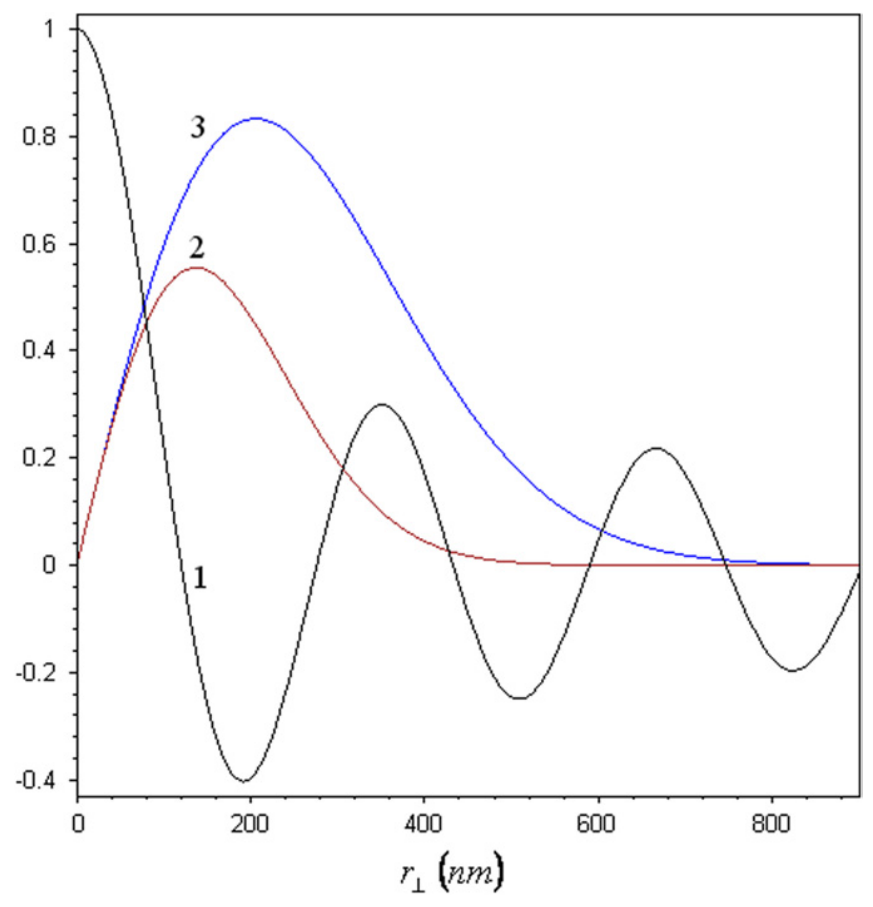

Figure 2. Dependences of the factors $I_{1}$ and $I_{2}$ on $r_{\perp}$. Curve (1) represents $I_{1}\left(r_{\perp}\right)$ for $q_{\perp}=2 \times 10^{-2} \mathrm{~nm}^{-1}$. Curves (2) and (3) are $0.03 \cdot I_{2}\left(r_{\perp}\right)$ for $\sigma=0.3 \mathrm{~nm}, l_{\perp}=200 \mathrm{~nm}$ (curve 2) and $l_{\perp}=300 \mathrm{~nm}$ (curve 3 ).

Usually the specular $\mathrm{x}$-ray scattering is described by the term $R_{1}$ only. The second term $R_{2}$ disappears for uncorrelated roughness and for smooth interfaces. Also the first term predominates in the case of very narrow slits in front of the detector, $\Delta \Omega \rightarrow 0$. As soon as the roughness is correlated and the detector slit has a finite size, both terms can be of the same order of magnitude. Moreover, for large $q_{z}, R_{2}$ transfers the main scattering contribution to the specular part. This leads to a broadening of the higher order Bragg peaks and to a slowing down of the Bragg intensity decay due to a difference in the dependences of the contributions $R_{1}$ and $R_{2}$ on $q_{z}$. The scattering intensity from the correlation part $R_{2}$ decays with $q_{z}$ more slowly than expected from the Debye-Waller like factor because it is partially compensated by the increasing multiplier $\left(\mathrm{e}^{\left[q_{z}^{2}\left\langle h_{n}\left(r_{\perp}\right) h_{m}(0)\right\rangle\right]}-1\right)$. Physically, correlated roughness and a finite detector slit opening, $\Delta \Omega \neq 0$, both result in the collection of diffuse scattering in addition to the specularly scattered radiation. Therefore in 'specular' scans both diffuse and specular scattering are collected simultaneously. This is well known in the literature and procedures have been described to separate specular from diffuse scattering [14].

Figure 3 shows a simulation of the combined reflection coefficient $R$ as well as the individual coefficients $R_{1}$ and $R_{2}$ as a function of the scattering angle $2 \theta$. The following values for the parameters were used, assuming a $[\mathrm{Fe}(2 \mathrm{ML}) / \mathrm{V}(16 \mathrm{ML})]_{30}$ multilayer on a $\mathrm{MgO}(001)$ substrate: $\lambda=0.154 \mathrm{~nm}$, $D=2.8 \mathrm{~nm}, d=2.51 \mathrm{~nm}, \sigma=0.3 \mathrm{~nm}, l_{\perp}=15 \mathrm{~nm}$, $l_{z}=15 \mathrm{~nm}, \Delta \Omega=3 \times 10^{-6} \mathrm{sr}$. The term $R_{1}$ is the main contribution for small $2 \theta$ values, whereas $R_{2}$ determines the intensity and the shape of higher order Bragg peaks.

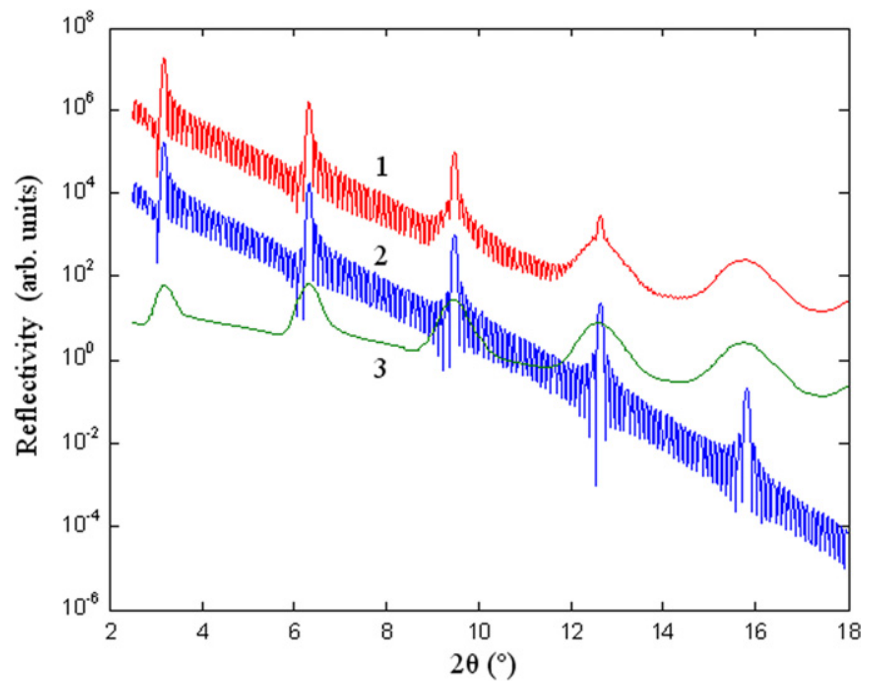

Figure 3. Calculated reflectivity for the multilayer $[\mathrm{Fe}(2 \mathrm{ML}) / \mathrm{V}(16 \mathrm{ML})]_{30}$. Total reflectivity $R$ (curve 1$)$, and contributions $R_{1}$ (curve 2 ) and $R_{2}$ (curve 3 ). Curve 1 is shifted for clarity.

According to equation (15) the term $R_{2}$ depends on the angular aperture of the detector. In our model the angular aperture is described by the solid angle $\Delta \Omega$, which is the simplest form of taking into account the instrumental resolution function.

Assuming that the roughness amplitude $\sigma$ does not depend on the layer number we obtain for $R_{1}$ from equation (14) oscillations between the Bragg peaks (Kiessig fringes), whereas the intensity decays with increasing $q_{z}$ due to the Debye-Waller factor. In contrast, the correlation function $\left\langle h_{n}\left(r_{\perp}\right) h_{m}(0)\right\rangle$ in the integral for $R_{2}$ (equation (15)) leads to the appearance of additional factors in the summation terms over the layers. Therefore the interference terms are destroyed and the film thickness oscillations between the Bragg peaks disappear. Furthermore, as the integrals in equation (15) increase with $q_{z}$, the disagreement between the terms in the summation over the layers also increases. This leads to a broadening of the high order Bragg peaks as seen in figure 3. The Kiessig fringes disappear in $R$, when the diffuse contribution $R_{2}$ crosses the specular contribution $R_{1}$.

To describe the specular scan the reflection coefficient $R$ is considered as a function of $q_{z}$. The interval where $I_{2}\left(r_{\perp}\right)$ is not close to zero depends on the magnitudes of $l_{z}$, $l_{\perp}$ and $q_{z}$. Results of the calculations for the same multilayer parameters as the ones used for figure 3 but for different combinations of the correlation lengths $l_{\perp}$ and $l_{z}$ are shown in figure 4. Variation of both correlation lengths changes the width of high order Bragg peaks. However the shape of these peaks depends on the $l_{z}$ and $l_{\perp}$ in different ways. Increase in $l_{z}$ leads to a more pronounced interference of the radiation reflected from different interfaces. As a result higher order Bragg peaks become more narrow. In contrast, increase in $l_{\perp}$ results in a broadening of the Bragg peaks, reduces the effect of the Debye-Waller factor and causes the Kiessig fringes to disappear. 


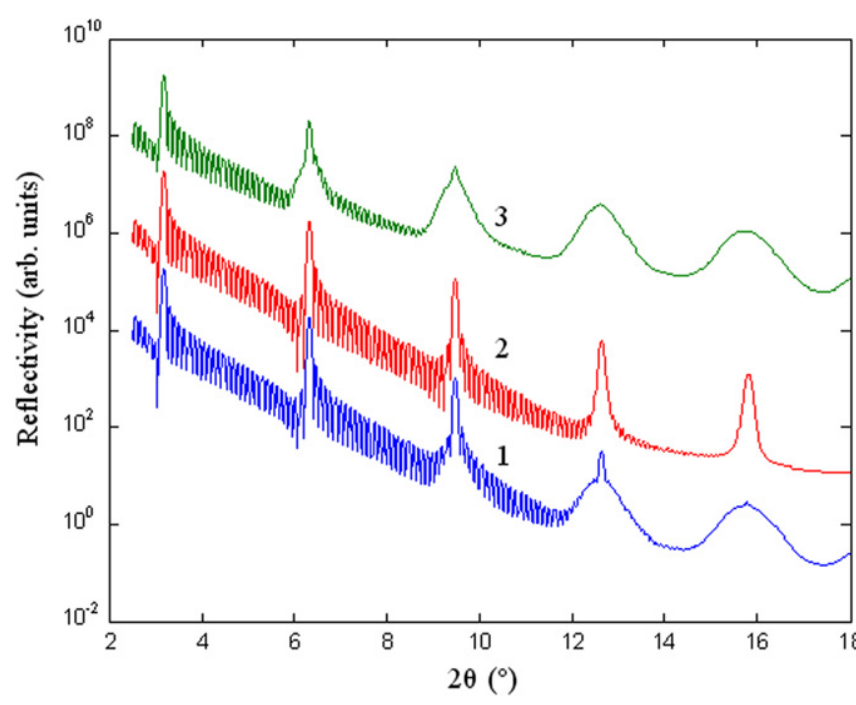

Figure 4. Calculated total reflectivity $R$ for the multilayer $[\mathrm{Fe}(2 \mathrm{ML}) / \mathrm{V}(16 \mathrm{ML})]_{30}$ and for different in- and out-of-plane correlation lengths: $1-l_{\perp}=15 \mathrm{~nm}, l_{z}=15 \mathrm{~nm} ; 2-l_{\perp}=15 \mathrm{~nm}$, $l_{z}=100 \mathrm{~nm} ; 3-l_{\perp}=100 \mathrm{~nm}, l_{z}=15 \mathrm{~nm}$. The curves are shifted vertically for clarity.

\section{Experimental procedures}

We will apply our approach for the description of x-ray scattering on two types of multilayers: $\mathrm{Fe} / \mathrm{V}$ and $\mathrm{CoFeB} / \mathrm{MgO}$ multilayes. The first type are among the most perfect multilayers that one can presently grow with unprecedented interface sharpness [15-17]. The second type is a combination of an amorphous metal and an oxide layer, which is of high interest for spin polarized electron tunnelling [18]. Depending on the growth procedures and annealing processes chosen, the layer thicknesses and interface sharpnesses in $\mathrm{CoFeB} / \mathrm{MgO}$ multilayers can also be very well defined [19]. However, in the example which we discuss here they are not.

$\mathrm{Fe} / \mathrm{V}$ multilayers are model systems for the investigation of interface magnetism [20], interlayer exchange coupling [16] and superconducting spin valves [21]. Especially the realization of the latter requires smooth interfaces to guarantee high interface transparency for the superconducting pair density function. We have sputter deposited a series of $[\mathrm{Fe}(2 \mathrm{ML}) / \mathrm{V}(16 \mathrm{ML})]$ multilayers with 30 repeats in a UHV sputter chamber with purified $\mathrm{Ar}$ sputtering gas at a pressure of $7 \mathrm{mbar}$ and a substrate temperature of $320^{\circ} \mathrm{C}$, following the procedures described in [15]. The multilayers were deposited on polished $\mathrm{MgO}\left(\begin{array}{ll}0 & 0\end{array}\right)$ substrates, starting with the vanadium layer and finishing with two ML of Fe. Finally the superlattice was capped in situ with a $50 \AA ̊$ palladium layer for protection against ambient oxidation. Room temperature XRR scans were taken at the wiggler beamline W1.1 using a photon energy of $E=8048 \mathrm{eV}(\lambda=1.5405 \AA)$ at the Hamburg Synchrotron Laboratory in Hamburg, Germany. The incident slit size after the monochromator was $2 \mathrm{~mm} \times 2 \mathrm{~mm}$ parallel and perpendicular to the scattering plane, respectively, placed at a distance of $1000 \mathrm{~mm}$ in front of the sample. After the sample we used two vertical slits (confining the beam in the scattering plane): at $300 \mathrm{~mm}$ behind the sample a slit of $0.5 \mathrm{~mm}$

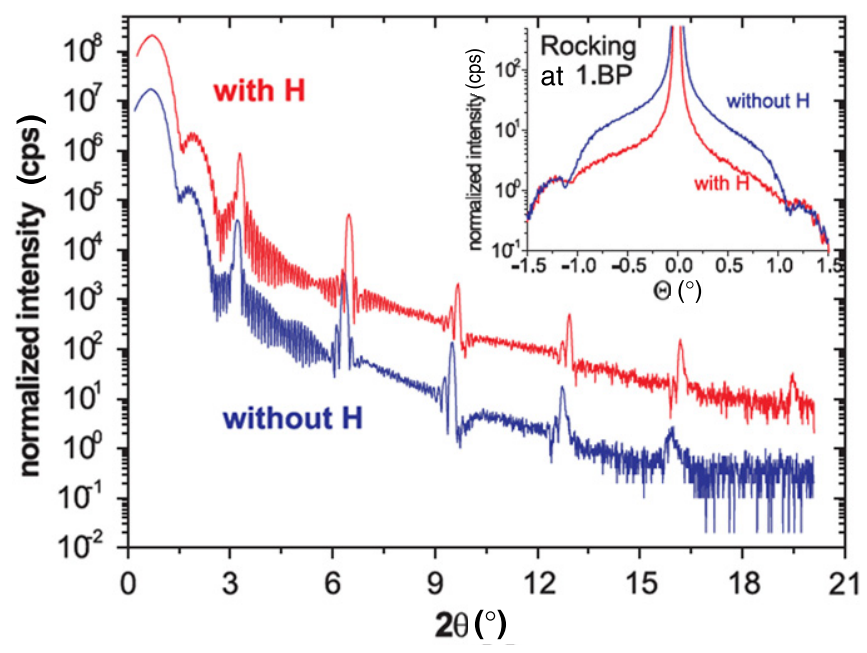

Figure 5. Experimental XRR data from a $[\mathrm{Fe}(2 \mathrm{ML}) / \mathrm{V}(16 \mathrm{ML})]_{30}$ multilayer. The superlattices were prepared without and with hydrogen assisted growth. The inset shows rocking scans across the first Bragg peak of the multilayers. From [17].

opening, and at $1200 \mathrm{~mm}$ behind the sample and in front of the detector a second slit also with a $0.5 \mathrm{~mm}$ opening was used. A more complete structural characterization of these multilayers is provided elsewhere [17].

The second set of multilayers $\mathrm{CoFeB} / \mathrm{MgO}$ was grown on $\mathrm{Si} / \mathrm{SiO}_{2}$ substrates by ion beam deposition of a $\mathrm{CoFeB}$ alloy target with the composition $\mathrm{Co}: \mathrm{Fe}: \mathrm{B}=60: 20: 20$. First a $100 \mathrm{~nm} \mathrm{SiO}{ }_{2}$ layer was grown on $\mathrm{Si}$, followed by a $2 \mathrm{~nm}$ thick $\mathrm{CoFeB}$ buffer layer and a $1.5 \mathrm{~nm}$ thick $\mathrm{MgO}$ buffer layer. Subsequently, the multilayer stack was deposited, consisting of 14 repeats of [CoFeB $3 \mathrm{~nm} / \mathrm{MgO} 1.5 \mathrm{~nm}$ ]. The $\mathrm{MgO}$ layer was obtained by oxidizing $\mathrm{Mg}$ in a natural flow of oxygen, $20 \mathrm{sccm}$ $\mathrm{O}_{2}$ flux for $120 \mathrm{~s}$. Finally the multilayer was capped with a $3 \mathrm{~nm}$ thick $\mathrm{CoFeB}$ layer followed by a $5 \mathrm{~nm}$ thick Ta layer. The total layer sequence together with the nominal layer thicknesses consists of $\mathrm{Si}$ substrate $/ \mathrm{SiO}_{2} 100 \mathrm{~nm} / \mathrm{CoFeB} 3 \mathrm{~nm} / \mathrm{MgO} 1.5 \mathrm{~nm}$ $[\mathrm{CoFeB} 3 \mathrm{~nm} / \mathrm{MgO} 1.5 \mathrm{~nm}] \times 14 / \mathrm{CoFeB} 3 \mathrm{~nm} / \mathrm{Ta} 5 \mathrm{~nm}$. The XRR measurements of the $\mathrm{CoFeB} / \mathrm{MgO}$ multilayers were performed at the beamline 9 of the DELTA Synchrotron source at the University of Dortmund [22]. An x-ray wavelength of $1.1272 \AA$, corresponding to an energy of $11 \mathrm{keV}$, was used. All $\mathrm{x}$-ray measurements were taken at room temperature. The slit size in front of the detector and at $1000 \mathrm{~mm}$ from the sample was $0.4 \mathrm{~mm}$ in the scattering plane and $7 \mathrm{~mm}$ perpendicular to the scattering plane, defining the resolution volume.

\section{Discussion}

Using our model described above, we provide simulations of the $\mathrm{x}$-ray data on the multilayer $[\mathrm{Fe}(2 \mathrm{ML}) / \mathrm{V}(16 \mathrm{ML})]_{30}$ on $\mathrm{MgO}(0001)$ substrates and from $[\mathrm{CoFeB} 3 \mathrm{~nm} / \mathrm{MgO} 1.5 \mathrm{~nm}]_{15}$ on $\mathrm{Si} / \mathrm{SiO}_{2}$ substrate.

In $\mathrm{Fe} / \mathrm{V}$ multilayers the interfaces can be made very smooth. Hydrogen assisted growth leads to an additional decrease in the interface roughness [17]. Therefore these multilayers are of considerable interest for testing our theory. Corresponding reflectivity scans from [17] are reproduced in figure 5. XRR scans from multilayers grown under hydrogen 


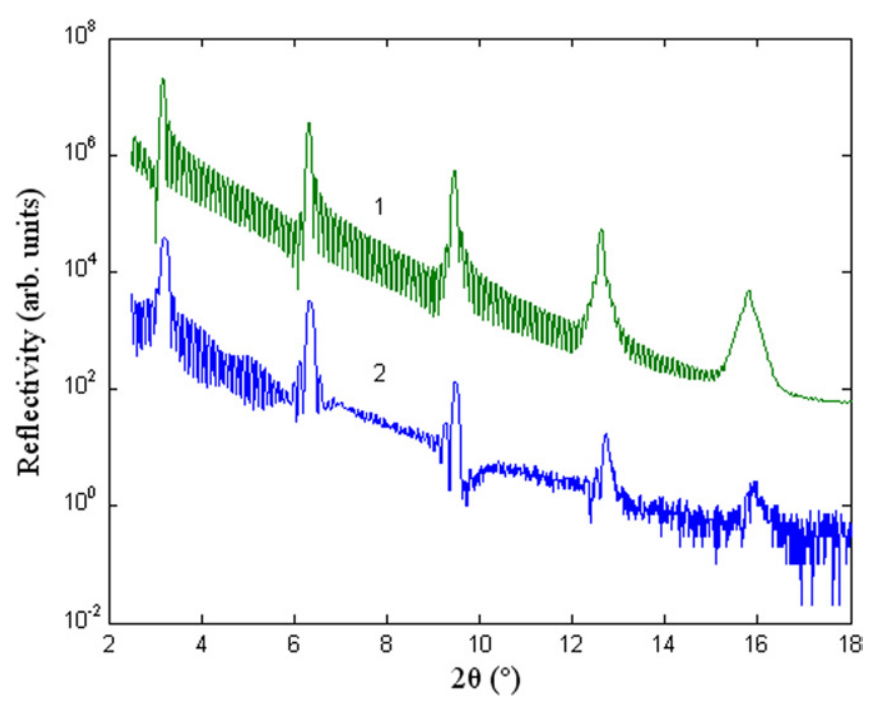

Figure 6. Comparison of experimental data (2) with results from simulations (1) for the multilayer $[\mathrm{Fe}(2 \mathrm{ML}) / \mathrm{V}(16 \mathrm{ML})]_{30}$ without hydrogen assisted growth.

assistance (top curve) show Fourier components (Bragg peaks) up to the sixth order, whereas XRR results from similar superlattices but grown without hydrogen assistance exhibit Fourier components only up to the fifth order (bottom curve).

The inset in figure 5 shows rocking scans across the first Bragg peak for samples without and with hydrogen assisted growth. It is worth noting that the slope of the diffuse part of this scan beneath the central peak is determined only by the value of the fractal (Hearst) parameter $H$ and by the in-plane correlation length $l_{\perp}$. In these scans it turns out that the slopes for both samples are practically the same. This is quite natural because the interaction of hydrogen with the metal atoms during epitaxial growth is rather local. It may decrease the interface roughness $\sigma$ but cannot affect the longrange correlation.

The experimental results from [17] for Fe/V multilayers grown without hydrogen assistance are shown in figure 6 together with the results of our simulation. For the simulation we used the parameters: $\lambda=0.154 \mathrm{~nm}, l_{\perp}=25 \mathrm{~nm}$, $l_{z}=25 \mathrm{~nm}, \Delta \Omega=3 \times 10^{-6} \mathrm{sr}, \sigma=0.23 \mathrm{~nm}$ for all interfaces. For the sample grown in a hydrogen atmosphere only the roughness parameter $\sigma$ was found to be slightly smaller. The simulation obviously catches the main features of the experimental scan, in particular the broadening of the fourth and fifth order Bragg peak.

In figure 7 we compare the experimental and theoretical results for $\mathrm{x}$-ray scattering from the $[\mathrm{CoFeB} 3 \mathrm{~nm} / \mathrm{MgO}$ $1.5 \mathrm{~nm}]_{15}$ multilayer. Here the parameters for the simulation are $\lambda=0.11272 \mathrm{~nm}, l_{\perp}=10 \mathrm{~nm}, l_{z}=12 \mathrm{~nm}$, $\Delta \Omega=3 \times 10^{-6} \mathrm{sr}, \sigma=0.5 \mathrm{~nm}$ for the substrate and $\sigma=0.7 \mathrm{~nm}$ for all other interfaces. One can immediately recognize that the theoretical simulations are in good agreement with the experimental results. It is important to note that the finite instrumental resolution (detector slit opening) for the 'specular' reflectivity scan provides information not only on the roughness amplitude $\sigma$ but also on the in- and out-ofplane correlation lengths.

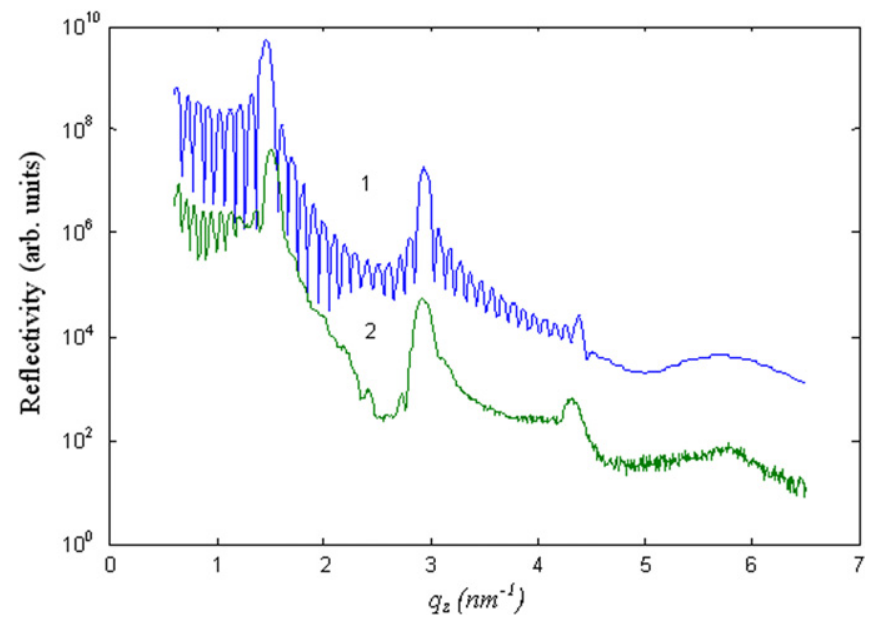

Figure 7. Comparison of experimental data (2) with results from simulations (1) for the multilayer $[\mathrm{CoFeB}(3 \mathrm{~nm}) / \mathrm{MgO}(1.5 \mathrm{~nm})]_{15}$.

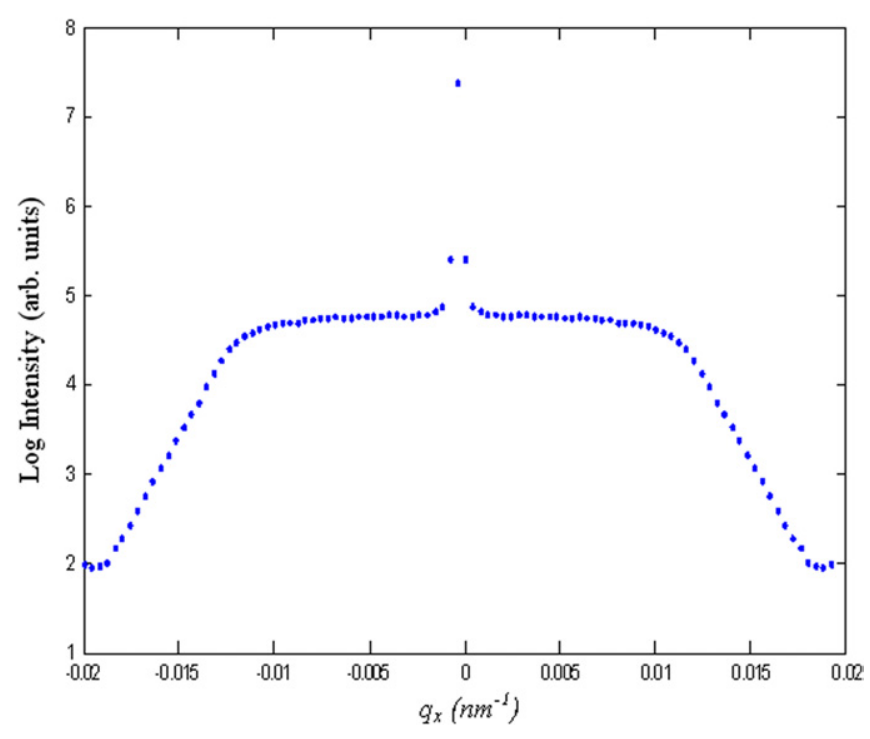

Figure 8. Experimental data on the transverse scan across the first Bragg peak of the multilayer $[\mathrm{CoFeB}(3 \mathrm{~nm}) / \mathrm{MgO}(1.5 \mathrm{~nm})]_{15}$.

The transverse scan across the first Bragg peak of the [CoFeB $3 \mathrm{~nm} / \mathrm{MgO} 1.5 \mathrm{~nm}]_{15}$ multilayer is shown in figure 8 . It is characterized by a central specular peak superimposed on a rather intense but almost flat diffuse scattering. The featureless diffuse scattering as a function of $q_{\perp}$ implies that the in-plane correlation length $l_{\perp}$ is below the limit achieved at the first Bragg peak, which was estimated to be on the order of $60 \mathrm{~nm}$. The same flat diffuse scattering without any noticeable slope also shows up at the Bragg peaks up to the third order. From this we estimate that the correlation length $l_{\perp}$ is less than $\left(60 / n^{2}\right) \mathrm{nm}(n=3) \approx 10 \mathrm{~nm}$. This value agrees well with the results obtained from the 'specular' scans.

For finite instrumental resolution (slit width) both correlation lengths $l_{z}$ and $l_{\perp}$ manifest themselves in the dependence of the specular reflectivity on the glancing angle $\theta$. According to our calculations and as illustrated in figure 4 these correlation lengths can be obtained by analysing the specular scattering data only. The experimental accuracy for determining the correlation lengths $l_{z}, l_{\perp}$, and for the interface 
roughness parameter $\sigma$ ill certainly increase by taking twodimensional intensity maps in the $q_{z}$ and $q_{\perp}$ directions. But even transverse scans across several Bragg reflections can help in determining the interfacial parameters.

\section{Concluding remarks}

In this contribution we have provided a theory for $\mathrm{x}$-ray scattering from thin films and multilayers at glancing angles within the Born approximation. In particular we have taken into account the finite instrumental resolution, which is always present in realistic experimental set-ups. This finite resolution causes specular and diffuse scattering to mix more and more with increasing scattering vector. Some unexpected XRR results can be understood on the basis of this mixing effect and, vice versa, can be used to determine the in- and outof-plane correlation lengths in addition to the interfacial roughness parameter. Our results can be summarized as follows:

1. For multilayer systems the broadening of high order Bragg peaks is a universal effect which is not connected with details of the sample preparation. It exists even for very smooth interfaces (see also [14]).

2. If the in-plane correlation length $l_{\perp}$ is small, transverse scans (rocking curves) cannot provide any information on its magnitude. However, in specular scans with finite slit opening this information is available. Therefore, it turns out that 'specular' scans can be more informative than diffuse scans even for the determination of the interfacial roughness parameter $\sigma$

3. Reflectivity scans using different slit widths (instrumental resolutions) can be useful to vary the contribution of the correlated roughness parameters $l_{\perp}$ and $l_{z}$ for the in- and out-of-plane correlation lengths, respectively, in the diffuse component $R_{2}$.

4. In diffuse scattering experiments the high order Bragg peaks are more sensitive to the in-plane roughness with small correlation length $l_{\perp}$

\section{Acknowledgments}

The authors are grateful to B Hjörvarsson, A Remhof for allowing them to reproduce the $\mathrm{X}$-ray scans shown in figure 5. This work was partly supported by the European Union's Marie Curie actions ULTRASMOOTH under contract MRTN-CT-2003-504462, by DFG-RFBR grant 06-02-04005, by SFB 491 and by RFBR grants 06-0801201 and 07-02-01065.

\section{References}

[1] Bontempi L, Depeero L E and Sanagaletti L 2000 Phil. Mag. B 8623

[2] Hsu C-H, Felcher G P, Liang K S Do, Noh Y and Sinha S K 2007 Thin Solid Films 5155529

[3] Fullerton E E, Schuller I K, Vanderstraeten H and Bruynseraede Y 1992 Phys. Rev. B 459292

[4] Stephanov S A, LLB and http://sergey.gmca.aps.anl.gov

[5] Philips X'pert: 2004 Reflectivity simulation software, version 1.1

[6] Tanner B K, Hase T P A, Fulthorpe B D, Clarke J, Luo G M, Halder S K, Rozatian A S H and Wilkins S B 2000 Mater. Res. Soc. Symp. Proc. 615 G2.1.1-G2.1.12

[7] Sinha S K, Sirota E B, Garoff S and Stanley H B 1988 Phys. Rev. B 382297

[8] Sanyal M K, Sinha S K, Gibaud A, Satija S K, Majrkzak C F and Homa H 1992 Surface X-Ray and Neutron Scattering (1992 Springer Proc. in Physics vol 61) ed H Zabel and I K Robinson $\mathrm{p} 91$

[9] Pietsch U, Holý V and Baumbach T 2004 High-Resolution $X$-Ray Scattering. From Thin Films to Lateral Nanostructures 2nd edn (New York: Springer)

[10] Sinha S K 1994 J. Phys. III France 41543

[11] Lee D R, Sinha S K, Haskell D, Choi Y, Lang J C, Stepanov S A and Srajer G 2003 Phys. Rev. B 68224409

[12] Lee D R, Sinha S K, Nelson C S, Lang J C, Venkataraman C T, Srajer G and Osgood R M 2003 Phys. Rev. B 68224410

[13] Schlomka J P, Tolan M, Schwalowsky L, Seeck O H, Stettner J and Press W 1995 Phys. Rev. B 512311

[14] Savage D E, Schimke N, Phang Y-H and Legally M G J 1992 J. Appl. Phys. 713282

[15] Isberg P, Hjörvarsson B, Wäppling R, Svedberg E B and Hultman L 1997 Vacuum 48483

[16] Hjörvarsson B, Dura J A, Isberg P, Watanabe T U, Udovic T J, Andersson G and Majkrzak C F 1997 Phys. Rev. Lett. 79901

[17] Remhof A, Nowak G, Liebig A, Zabel H and Hjörvarsson B 2006 J. Phys.: Condens. Matter 18 L441-5

[18] Moodera J S, Kinder L R, Wong T M and Meservey R 1995 Phys. Rev. Lett. 743273

[19] Miaoa G-X, Chetry K B, Gupta A, Butler W H, Tsunekawa K, Djayaprawira D and Xiao G 2006 J. Appl. Phys. 9908 T305

[20] Scherz A, Wende H, Poulopoulos P, Lindner J, Baberschke K, Blomquist P, Wäppling R, Wilhelm F and Brookes N B 2001 Phys. Rev. B 64180407

[21] Westerholt K, Sprungmann D, Zabel H, Brucas R, Hjörvarsson B, Tikhonov D A and Garifullin I A 2005 Phys. Rev. Lett. 95097003

[22] Krywka C, Paulus M, Sternemann C, Volmer M, Remhof A, Nowak G, Nefedov A, Pöter B, Spiegel M and Tolan M 2006 J. Synchrotron Radiat. 138 